\title{
Dynamical Evolution of Planetary Systems
}

\author{
Alessandro Morbidelli
}

\begin{abstract}
Planetary systems can evolve dynamically even after the full growth of the planets themselves. There is actually circumstantial evidence that most planetary systems become unstable after the disappearance of gas from the protoplanetary disk. These instabilities can be due to the original system being too crowded and too closely packed or to external perturbations such as tides, planetesimal scattering, or torques from distant stellar companions. The Solar System was not exceptional in this sense. In its inner part, a crowded system of planetary embryos became unstable, leading to a series of mutual impacts that built the terrestrial planets on a timescale of $\sim 100 \mathrm{My}$. In its outer part, the giant planets became temporarily unstable and their orbital configuration expanded under the effect of mutual encounters. A planet might have been ejected in this phase. Thus, the orbital distributions of planetary systems that we observe today, both solar and extrasolar ones, can be different from the those emerging from the formation process and it is important to consider possible long-term evolutionary effects to connect the two.
\end{abstract}

\section{Introduction}

This chapter concerns the dynamical evolution of planetary systems after the removal of gas from the proto-planetary disk. Most massive planets are expected to form within the lifetime of the gas component of protoplanetary disks (see chapters by D'Angelo and Lissauer for the giant planets and by Schlichting for Super-Earths) and, while they form, they are expected to evolve dynamically due to gravitational interactions with the gas (see chapter by Nelson on planet migration). However, although often forgotten, the dynamical evolution of planetary systems is not over once the gas disappears. In the next section will review the observational evidences

Alessandro Morbidelli

Université Côte d' Azur, CNRS, Observatoire de la Côte d' Azur, Boulevard de l' Observatoire, CS 34229, 06304 Nice Cedex 4, France, e-mail: hdeeg@iac.esmorby@oca. eu 
for relevant post-gas evolution. Most planetary systems that we observe are around main-sequence stars and therefore post-gas evolution needs to be taken into account if we want to explain their current orbital characteristics. Post-gas evolution is essentially due to dynamical instabilities in the planetary system and/or interactions with a remnant planetesimal disk. We will discuss planetary systems becoming unstable by themselves, i.e. without interactions with planetesimals or stellar companions, and the outcome of these instabilities. Then, we will discuss processes that may destabilize a system which would remain otherwise stable. These include interactions with the disappearing gas-disk, with a remnant planetesimal disk, with a stellar companion or tidal interactions with the host star. The final section will provide a brief summary.

\section{Observational evidence for post-gas evolution of planetary systems}

Let's start from the Solar System, the planetary system that we know the best. There are several populations of small bodies that have a very different orbital structure than the one expected for planetesimals formed in a proto-planetary disk. In fact, the asteroid belt, the Kuiper belt and the Trojans of Jupiter and Neptune have excited eccentricities and inclinations, whereas pristine planetesimal disks should have quasi-circular, coplanar orbits. Clearly, strong perturbations affected all these populations, such as close encounters or resonant interactions with planets, possibly some of which are no longer in the system. The irregular satellite systems around all giant planets and the existence of the Oort cloud of comets also offer evidence for a violent dispersion of the original planetesimal disk. It is important to realize that the dynamical excitation and dispersal of planetesimals should have occurred after the removal of gas from the proto-planetary disk, because gas drag (Adachi et al., 1976) or gas-dynamical friction (Grishin and Perets, 2015) would have been very effective in re-circularizing the planetesimals orbits and in bringing them back onto the disk's mid-plane (see for instance Brasser et al., 2007). Because the asteroids, Kuiper belt objects and Trojans are stable with today's orbital configuration of the giant planets, this is strong evidence that the orbits of the planets were originally different and evolved to the current configuration sometime in the post-gas era of the Solar System.

Studies of planet migration (see chapter by Nelson), suggest what orbital configuration the giant planets should have had at the end of the gas-disk lifetime. In fact, because of its larger mass, Jupiter migrates towards the Sun more slowly than Saturn, Uranus and Neptune. Thus the giant planets should have had convergent migration relative to each other and the natural outcome of this process is that their orbits locked in mutual mean motion resonances, where the orbital periods of the planets are in interger numbers ratio (Morbidelli et al., 2007). Thus, the giant planets had to evolve from this primordial multi-resonant configuration to the current one. They could have done so only after gas removal, because otherwise gas-driven 
migration would have brought them back into resonance. The so-called Nice model (Morbidelli et al., 2007; Levison et al., 2011; Batygin et al., 2012; Nesvorny and Morbidelli, 2012) explains that this change in orbital configuration of the giant planets happened during a phase of dynamical instability and shows how this instability sculpted the small body populations, as briefly reviewed in Sect. 4 .

The extrasolar planets provide evidence that evolution in orbital configuration after gas-removal is the norm rather than an exception for the giant planets of the Solar System. The extrasolar giant planets are typically found on eccentric orbits. The best explanation for the statistical distribution of their orbital eccentricities is that the observed planets were initially part of a multi giant-planet system which became violent unstable, some of the original planets being ejected or tossed onto undetectable long-period orbits (Ford and Rasio, 2008; Juric and Tremaine, 2008; Chaterjee et al., 2008, Beauge and Nesvorny, 2012). These instabilities should have occurred after gas dissipation because otherwise the planet-disk interactions would have re-stabilized the planetary orbits in a new compact configuration with loweccentricities (Lega et al., 2013).

In contrast with extrasolar giant planets, super-Earths typically have orbits with small eccentricities. Nevertheless there are two lines of evidence arguing that the vast majority of super-Earth systems (probably more than 90\%) experienced postgas instabilities as well (Izidoro et al., 2017).

The first line of evidence is that super-Earths should have been in multi-resonant configurations at the end of the gas-disk lifetime, while the observed distribution of orbital periods of adjacent super-Earths shows a broad distribution with no preference for ratios of integer numbers (i.e. resonances). In fact the typical spacing between super-Earths tends to be around 20 mutual Hill-radii (see sect. 3 for a definition), whatever period ratio that implies ( $\mathrm{Pu}$ and $\mathrm{Wu}, 2015)$. Only very few systems are confirmed to be in resonant configurations (e.g. Mills et al., 2016). The reason for multi-resonant configurations in the disk of gas comes from the realization that the existence of so many planets on orbits with small semi major axis (statistical studies suggest that about 1 out of 3 solar type stars host at least one super-Earth with orbital period shorter than 100 days: Mayor et al. 2011; Howard et al. 2012; Fressin et al. 2013; Petigura et al. 2013) implies that planets cannot migrate all the way into the central star. Most likely, their migration is stopped at a disk's inner edge (Masset et al., 2006). This edge could correspond to the outer radius of a magnetic cavity (Ferreira and Pelletier, 1995; Armitage, 2010) or to the transition between an MRI active, low-density region of the disk and a low-viscosity, high-density region (Gammie 1996; Fromang et al. 2002). Super-Earth migration can also be stopped at other locations of the disk where density or temperature gradients are particularly steep (Hasegawa and Pudritz, 2010, 2011; Bitsch et al., 2015). In these conditions a system of migrating planets necessarily experiences convergent migration from the moment the first of them reaches the planet trap", where its inward migration is stopped. Locking in mutual mean motion resonances should then be the natural outcome. This is expected independent of whether the super-Earths grow in the inner disk (Ogihara et al., 2015) or migrate from beyond the snowline (Cossou et al., 2014). The same outcome is expected also in the inside-out planet formation 
model of Chatterjee and Tan (2014), in which planets form in sequence from the accumulation of dust at the disks inner edge.
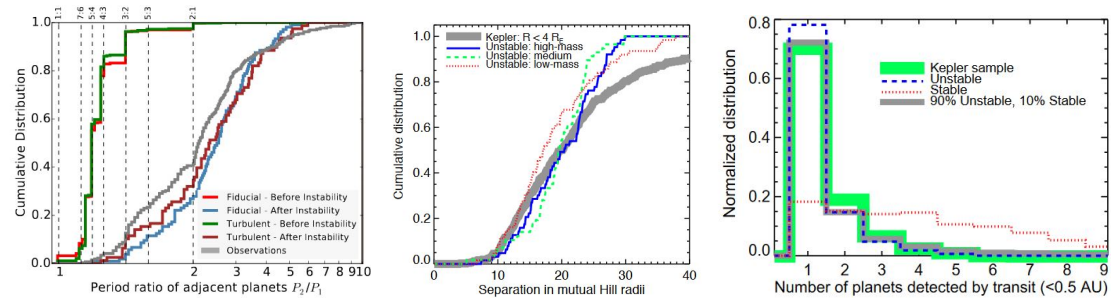

Fig. 1 Left: The cumulative distribution of period ratios for adjacent planets. The gray curve shows the observed distribution. The green and red curves are for the system at the disappearance of the disk of gas, before their dynamical instability. The brown and blue curves show the distribution after the dynamical instability. The green and brown distributions are from simulations which included the effects of turbulence in the disk of gas, and are essentially identical to those (red, blue) not accounting for turbulence. Center: The cumulative distribution of separations of adjacent planets in units of mutual Hill radii $R_{H}$. The gray curve shows the observed distribution and the blue, green and red curves show the distributions obtained after the instability, broken-down by planet mass-range. Right: The normalized histogram of number of planets detected by transit in a given system. The green histogram depicts the distribution observed by Kepler; the blue histogram shows the distribution expected for systems that underwent instability and the red histogram that of system that did not evolve in the aftermath of gas-removal. The grey histogram fitting the observed distribution is obtained assuming that $90 \%$ of observed system underwent instability. From Izidoro et al. (2017)

The second line of evidence for instabilities is that more than $50 \%$ of the observed systems of transiting super-Earths are made of a single planet. Either these planets are truly alone, which would be surprising, or there must be enough dispersion in the inclinations of the planets that only one of them is transiting (Fang and Margot, 2012; Johansen et al., 2012) $\sqrt{1}$ The inclination damping in the gas-disk should produce co-planar systems (Cresswell and Nelson, 2008), so the inclination dispersion needs to have been acquired after gas-dispersal.

Izidoro et al. (2017) showed that the observed distribution of orbital period ratios between adjacent planets (Fig. 1] left), as well and that of orbital separation in terms of mutual Hill radius (Fig. 1, center), are very well reproduced by systems that are in resonant chains at the time the gas is removed from the system and that become unstable afterwards. The systems that experience this post-gas instability also reproduce the statistics of the number of planets detected by the satellite Kepler around individual stars (Fig. 11, right). Thus, post-gas dynamical instabilities should have been the norm in super-Earth systems.

A final indication for a relevant evolution of planetary systems after gas dissipation comes from the observations of debris disks with hot dust around main sequence

\footnotetext{
${ }^{1}$ Radial-velocity observations could in principle solve this question because they can detect also non-transiting planets. Unfortunately the possibilities of RV follow-up of the systems discovered by the satellite Kepler are limited because the stars observed by Kepler are too faint
} 
stars, such as $\eta$ Corvi (Lisse et al., 2012; Marino et al., 2017) or Vega (Marboeuf et al., 2016). The dust produced in the vicinity of these stars cannot be sustained by the collisional grinding of local planetesimal populations because these populations could not remain massive for long enough. Instead, the best explanation is that planetesimals from a distant disk are scattered inwards as comets by planets (Wyatt et al., 2007; see also the chapter by Wyatt in this book). The scattering of a large number of planetesimals for a long time requires that planets are currently migrating through the planetesimal disk due to the scattering process itself (Bonsor et al., 2014).

Prompted by all these observational indications that evolution of planetary systems in the aftermath of gas removal is far from trivial, in the next sections we will examine the processes causing this evolution and their consequences.

\section{Planetary systems becoming unstable by themselves}

A first analysis of stability of two adjacent planets on circular orbits was done by Gladman (1993) in the framework of the Hill problem. He found that the planets are Hill-stable (i.e. stable against mutual close approaches for all times) if their orbital separation exceeds $2 \sqrt{3}$ mutual Hill radii $\left(R_{H}\right)$, where $R_{H}=\left[\left(m_{1}+\right.\right.$ $\left.\left.m_{2}\right) / 3 M_{\text {star }}\right]^{1 / 3}\left(a_{1}+a_{2}\right) / 2, m_{1}$ and $m_{2}$ are the masses of the two planets with semi major axes $a_{1}$ and $a_{2}$, and $M_{\text {star }}$ is the mass of the star (notice that Gladman used a different definition for the mutual Hill radius and therefore his numerical coefficient is slightly different). The instability of planets with orbital separation smaller than this threshold has been interpreted by Deck et al. (2013) as the consequence of the fact that resonances become wider than their mutual separation; thus they overlap, generating large-scale chaos (Chirikov, 1979). For more than two planets, no stability criteria can be found analytically. Thus, Chambers et al. (1996) used numerical simulations. They found that systems with orbital separations between adjacent planets smaller than 10 mutual Hill radii are always unstable, with an instability time whose logarithm

$$
\log t \sim b \Delta+c
$$

where $\Delta$ is the mutual separation in units of $R_{H}$ and $b$ and $c$ are constants. The constant $b$ is weakly dependent on the number of planets and becomes independent on the planetary masses (assumed equal for all planets) if $\Delta$ is rescaled by a quantity proportional to $m^{1 / 4}$, instead of $R_{H}$.

This work has been recently revisited by Obertas et al. (2017), who confirmed the previous results showing a linear trend in the initial planet spacing between 3 and $8 R_{H}$ and the logarithm of the stability time. Moreover, investigating thousands of simulations with spacings up to $13 R_{H}$, they revealed distinct modulations superimposed on this relationship in the vicinity of first and second-order mean motion 
resonances (corresponding to period ratios $(n+1) / n$ and $(n+2) / n$, for integer $n$, respectively) of adjacent planets (Fig. 2).

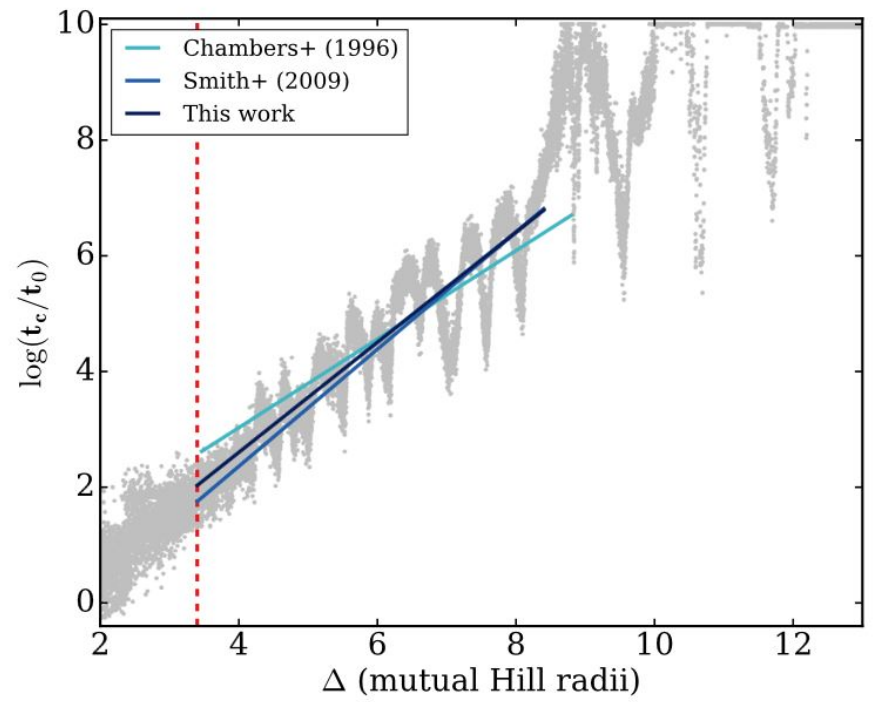

Fig. 2 The instability time as a function of mutual separation between adjacent planets in multipleplanet systems, normalized in units of mutual Hill radius (Obertas et al., 2017). The colored solid lines compare the mean relationships found in Obertas et al., (2017), Chambers et al. (1996) and Smith and Lissauer (2009). The vertical dashed line shows the limit of Hill stability for a pair of planets, from Gladman (1993).

Focusing specifically on first order mean motion resonances, Matsumoto et al. (2012) found a critical number of planets in resonant chains, beyond which stability is not possible after gas removal. In the unstable case, the instability time is similar to the non-resonant case (i.e. formula (1)). This critical number $N_{c r i t}$ depends on the resonant configuration. At equal planetary masses, compact resonant chains (e.g. each planet being in the 8:7 resonance with its neighbor) have $N_{c r i t}$ smaller than more spaced resonant chains (e.g. in 6:5 resonances); the values of $N_{\text {crit }}$ are respectively 3 and 8 for planet masses of $10^{-5}$ stellar masses. In fact, $N_{\text {crit }}$ is a growing function of the mutual separation in $R_{H}$ (although not a simple analytic one) and therefore, for a given resonant chain, it can increase substantially if the planetary masses are reduced.

Like the results of Chambers et al. and Obertas et al., those of Matsumoto et al. are also purely numerical, and there are currently no theoretical explanations for these empirical stability laws. For the simpler case of two planets in a firstorder resonance, Pichierri et al. (2018) find that the instability occurs when the masses are large enough that their mutual Hill radius becomes larger than $\sim 1.3$ times the minimal approach distance that the planets have from each other, if they are placed at the center of the resonance. This factor of 1.3 is much larger than the 
one $(1 /(2 \sqrt{3}) \sim 0.3)$ for non-resonant orbits, but it is found to decrease with the amplitude of libration in the resonance. It will be interesting to attempt a generalization of this result to a multi-planet resonant chain.

Properly understood or not, the results quoted above show that systems in which planets are too close to each other relative to their mutual Hill radius become unstable on some characteristic timescale after gas-removal, without the need of any external perturbation.

However, the simulations of Izidoro et al. (2017), which build the resonant chains among super-Earths through a consistent modeling of the accretion and migration processes affecting these planets, show that only $50 \%$ of the systems become unstable by themselves after gas removal. This is insufficient with respect to the estimated fraction of unstable systems $(>90 \%)$ required to fit the observed distributions (Izidoro et al., 2017). Thus, some external perturbations are needed in order to fully explain the observations, and these will be discussed in the next section.

Once a planetary system becomes unstable, in absence of damping exerted by the gas or planetesimal dynamical friction, the fate of the system depends on the so-called Safronov number. This is

$$
\Theta=\frac{v_{e s c}^{2}}{2 V_{o r b}^{2}},
$$

where $v_{\text {esc }}$ is the escape velocity from the planets and $V_{\text {orb }}$ is their orbital velocity (Safronov, 1972). If this number is larger than one, the close encounters among the planets are likely to lead to the ejection of some of the bodies until a new stable configuration is achieved. In fact, mutual scattering tend to give the planets a velocity dispersion of order $v_{e s c}$. If the latter exceeds the escape velocity from the potential well of the star, which is equal to $\sqrt{2} V_{\text {orb }}$, an ejection from the system is inevitable. If instead the Safronov number is smaller than unity, collisions among the planets are more likely. In this case, the reduced number of planets (albeit with larger masses due to the merging collisions) also eventually leads to a new stable configuration.

In the case of giant planets in the outer part of the disk, $\Theta$ is typically larger than 1 and therefore planets are ejected when instability occurs. This was probably the fate of extrasolar giant planet systems, leaving behind a single detectable planet on eccentric orbit (Ford and Rasio, 2008; Juric and Tremaine, 2008; Chaterjee et al., 2008). The instability that characterized the giant planets of our Solar System is also likely to have ejected at least one planet (Batygin et al., 2012; Nesvorny and Morbidelli, 2012), while Uranus and Neptune have been saved by the dynamical friction exerted by the trans-Neptunian planetesimals (see next section). For close-in super-Earth systems, instead, $\Theta<1$ and, therefore, planets merge in collisions when they become unstable (Izidoro et al., 2017). An important point made in Izidoro et al. is that collisions may happen before the velocity dispersion has the time to reach the asymptotic value $v_{\text {esc }}$, so that, when the number of planets decreases enough to achieve a stable configuration, the system can remain frozen with an eccentricity and inclination excitation smaller than expected. 
The classic model of formation of terrestrial planets (Chambers and Wetherill, 1998; Agnor et al., 1999; Chambers, 2001) in the Solar System is based on the selfinstability of the inner solar system planetary embryos after gas-removal. In fact, the planetary embryos formed in the inner part of the proto-planetary disk by oligarchic growth are expected to have been approximately Mars-mass and separated by 510 mutual Hill radii (Kokubo and Ida, 2000). Due to their large number and short spacing, in view of the results of Chambers et al. (1996) the system of planetary embryos becomes naturally unstable on a timescale of $10^{5} \mathrm{y}$ after gas removal. At $1 \mathrm{AU}$, the Safronov number for a Mars-mass body is 0.013 and for an Earth-mass body it is 0.067 . Thus the outcome of the instability is mutual collisions of planetary embryos, building the Earth and Venus through a series of giant impacts.

\section{Evolution of planetary systems under external perturbations}

This section discusses some processes that can favor the onset of planet instabilities after gas removal.

\section{Interactions with the disappearing gas-disk}

Turbulence in the disk may prevent capture of the planets deeply into resonances (Adams et al., 2008; Batygin and Adams, 2017), favoring their eventual orbital instability. As it was said before, instabilities within the disk of gas are likely to be recovered due to the damping action of the disk (Lega et al., 2013). Thus, for turbulence to be a trigger of an instability that can leave a permanent imprint on the final orbital structure of the system, the disk has to remain strongly turbulent until it disappears. It is even possible that disks become turbulent near the end of their lifetime. In fact, with increasing optical depth the ionization of gas in the midplane due to the stellar and interstellar irradiation becomes possible, potentially activating the magneto-rotational instability. However, if the density of gas is weak, the effects of turbulence may be insufficient to extract the planets from the core of mean motion resonances where they are likely to have been captured when the disk was still laminar (Deck and Batygin, 2015). Izidoro et al. (2017) modeled disk turbulence following the recipe of Laughlin et al. (2004) and Baruteau and Lin (2010) and they concluded that turbulence does not enhance the probability that a system of super-Earths becomes unstable. This result, however, may depend on the assumed scaling of turbulence strength with heliocentric distance. It should be said that it is now questioned whether disks can be turbulent; recent studies find that non-ideal magneto-hydrodynamic effects may quench turbulence in most of the disk (e.g. Bai and Stone, 2013).

As the disk disappears, the magnetic cavity is likely to expand because the balance between the magnetic torque and the viscous torque (that sets the disk's trunca- 
tion) occurs farther and farther from the star as the disk's density is reduced (Ferreira and Pelletier, 1995; Armitage, 2010). Liu et al. (2017) considered the effects that the expansion of the magnetic cavity has on a system of resonant super-Earths near the disk's inner edge. They find that if the expansion of the cavity is slow enough and the disk beyond the cavity is still massive enough compared to the planets, the planet at the disk's edge remains locked with the edge and migrates outwards with it as the cavity expands. Eventually, the inner planet does not manage to follow the radial motion of the disk's edge and it ends up in the cavity, where its migration stops. The receding edge can then entrain outwards for a while the second planet and so forth. The result is that the planets are extracted from their original resonance and deposited on quasi-circular and co-planar orbits with wider and non-resonant orbital periods. If this can explain the lack of preference for resonant ratios seen in the Kepler data, this process alone cannot fully explain the data: for instance, the fraction of stars with multiple transiting planets would be too large because of the coplanar geometry of the final planetary systems (Izidoro et al., 2017). However, as planets departure from their original resonance, they can cross new resonances which can excite their orbital eccentricities enough to trigger orbital instabilities, as in the case of the Nice model (Tsiganis et al., 2005; Morbidelli et al., 2007). How frequently an instability can occur in this process has not been quantified.

Another changing property of a disappearing disk is its vertical aspect ratio. In the inner part of the disk the dominant heating mechanism which sets the aspect ratio is viscous heating, which decreases with decreasing accretion rate onto the central star (Bitsch et al., 2015). As the aspect ratio of the disk decreases, the eccentricity of resonant planets undergoing damping from the disk also decreases (see e.g. Xu and Lai, 2017). With decreasing eccentricity the frequency of libration of resonant planets increases (Batygin and Morbidelli, 2013). This may cause instabilities due to the passage through commensurabilities among the libration periods of multiple resonant planets or between libration and synodic periods.

\section{Interaction with remnant planetesimals}

The removal of the gas leaves behind the planetesimals. The interaction between planets and planetesimals can be neglected as long as there is a lot of gas in the system, but it becomes predominant once the gas-disk is substantially depleted (Capobianco et al., 2011). The scattering of planetesimals by a planet changes the orbit of the planet by the action-reaction principle. A single planet embedded in a planetesimal disk typically migrates inwards, because of a scattering bias, discussed in Kirsh et al (2009), that favors the scattering of planetesimals in the outwards direction. However, two (or more) planets on nearby orbits typically migrate in divergent directions, the outer planet(s) moving outwards and the inner one inwards, because the outer planet acts as a conveyor belt, transfering planetesimals from the outer disk to the inner planet (Fernandez and Ip, 1984; see Levison et al., 2007 for a review of planetesimal-driven migration). 
In the Nice model, the giant planets are initially in a mean-motion resonant chain. The scattering of planetesimals by the planets extracts the latter form their original resonances through divergent migration. The crossing of other higher-order resonances gives the planets enough eccentricity excitation to trigger a global instability (Morbidelli et al., 2007, Fig. 33. If the planetesimal disk is far enough so that the planets cannot scatter them, the secular planet-planetesimal interactions can still modify the resonant orbits of the planets and enhance their eccentricities until a global instability follows (Levison et al., 2011). However, the flux of dust generated by the distant planetesimal disk due to its slow collisional grinding can also drive divergent migration of the planets (Deienno et al., 2017). In the instability phase, the planets have mutual close encounters. These excite the eccentricities and inclinations of the planetary orbits and drive the planets away from each other much more violently than what the sole scattering of planetesimals could do. However, the planetesimals still play a fundamental role: by exerting dynamical friction on the planets, they eventually damp the planetary eccentricities and inclinations, allowing the planets to recover a stable configuration with moderately excited orbits (Tsiganis et al., 2005; Morbidelli et al., 2007; Nesvorny and Morbidelli, 2012; see Fig. 3). In this process the planetesimals are violently dispersed and only those landing in stable niches of the orbital space survive. Thus, reproducing the current orbital structure of the surviving planetesimal populations is a crucial diagnostic of an instability model, possibly more than the final orbits of the planets themselves. In this respect, the Nice model is extremely successful and it is currently unchallenged by alternative models. In fact, it explains the capture and the orbital distribution of the Trojans of Jupiter (Nesvorny et al., 2013) and of Neptune (Nesvorny and Vokrouhlicky, 2009) and of the irregular satellites of the giant planets (Nesvorny et al., 2007), the final orbital sculpting of the asteroid belt (Morbidelli et al., 2010; Roig and Nesvorny, 2015; Deienno et al. 2016) and of the different components (cold, hot) of the Kuiper belt (Nesvorny, 2015a,b), as well as the origin of the Oort cloud and scattered disk (Brasser and Morbidelli, 2013; Nesvorny et al., 2017).

Chatterjee and Ford (2015) proposed that divergent migration due to planetesimal scattering is also the dominant process that extracts super-Earths form their original resonant chain. As already commented above concerning the cavity expansion mechanism of Liu et al. (2017), simply extracting the planets from the resonances on circular and co-planar orbit would not be sufficient to explain the observations. A dynamical instability is needed to produce the inclination excitation deduced from the frequency of multiple transiting-planets (Izidoro et al., 2017). But we have seen that divergent migration can also trigger instabilities. However, it is unclear what are the distribution and the total mass of planetesimals in the vicinity of super-Earths at the disappearance of the gas-disk. If the super-Earths migrated from larger distances, it is likely that their broad neighborhood was substantially depleted of planetesimals during the migration phase. If this is true, the remnant planetesimals would not carry enough mass to be able to change substantially the planets' orbits in the aftermath of gas removal. Notice that, if the Nice model is correct, this was not the case of the Solar System: the planetesimal disk was just a few AUs beyond Neptune (which was much closer to the Sun than its current orbit, presumably around 12-15 AU). 


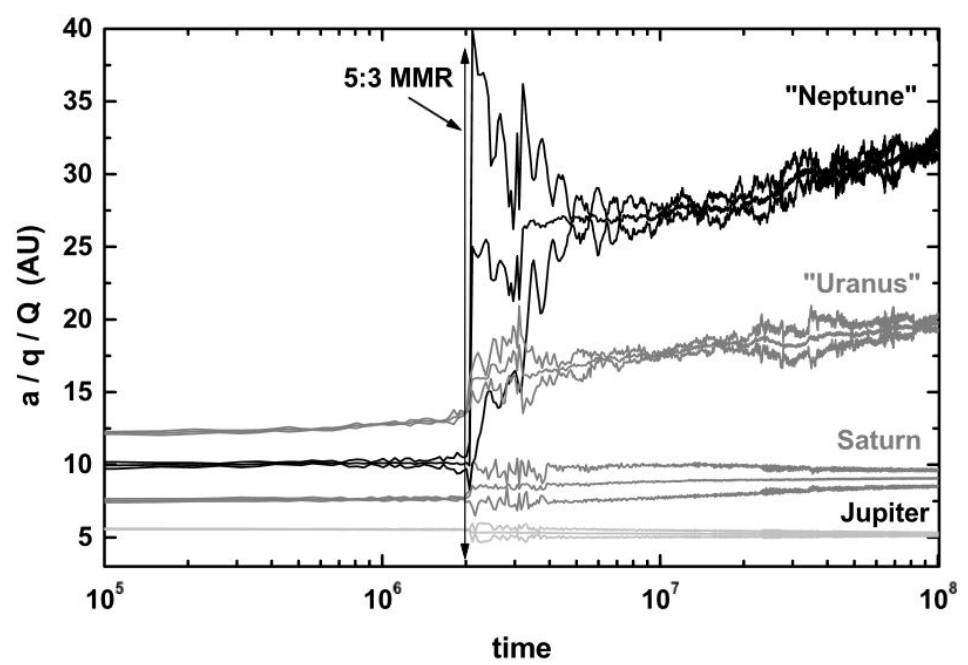

Fig. 3 A possible evolution of the giant planets of the Solar System in the Nice model (from Morbidelli et al., 2007). Each planet is represented by three curves, depicting the perihelion distance $q$, the semi major axis $a$ and the aphelion distance $Q$, plotted vs. time (in years). Initially the planets are in the multi-resonant configuration 9:6:4:3 (Jupiter doing 9 revolutions while Saturn does 6, Uranus 4 and Neptune 3). Planetesimal scattering forces the planets to migrate in divergent directions and leave the original resonances. When Jupiter and Saturn cross the 5:3 resonance, the excitation of their eccentricities triggers a global instability, followed by a phase of close encounters between pairs of planets. Uranus and Neptune are pushed outwards on large eccentricity orbits and are their eccentricities are finally damped by the dynamical friction exerted by the dispersing planetesimal disk. This version of the Nice model starts and ends with 4 giant planets. But the are other versions, even more successful in terms of final planetary orbits, with initially three Neptune-mass planets, of which one is ejected during the instability (Batygin et al., 2012; Nesvorny and Morbidelli, 2012).

Probably, the reason for this was that the giant planets did not migrate inward significantly, or even migrated outwards, during the gas-disk phase, most likely as a consequence of the resonant interactions between Jupiter and Saturn (Masset and Snellgrove, 2001; Walsh et al., 2011).

\section{Tidal interactions with the central star}

Planets on orbits close to the central star undergo a substantial orbital evolution due to tidal dissipation. The dissipation in the star drives primarily the orbital migration of the planet, whereas the dissipation in the planet damps primarily its orbital eccentricity (Goldreich and Soter, 1966). For planets in first order mean motion resonances, the eccentricity damping forces the planets to migrate away from each other, due to the shape of the resonant locus in the eccentricity vs. semi major axis plane 
(Papaloizou and Terquem, 2010; Lithwick and Wu, 2012; Batygin and Morbidelli, 2013b). Thus, this process is equivalent to that of divergent migration discussed above.

The tidal migration of a planet due to the dissipation in the star has a direction that depends on the ratio between the rotation rate of the star and the orbital rate of the planet. If the star rotates faster than the orbital motion of the planet, the planet migrates outwards (like the case of the Moon, relative to the Earth); in the opposite case the migration is inwards. For two planets in resonance, the tidal effect is much stronger on the inner planet. If the latter migrate inwards, we have again a case of divergent migration. Otherwise we have a case of convergent migration. This case is more interesting: the resonant configuration prevents the period ratio $P_{2} / P_{1}\left(P_{2}\right.$ being the orbital period of the outer planet) to become smaller than the resonant ratio and in turns converts convergent migration into eccentricity excitation. This eccentricity excitation is not unbound because of the tidal damping on the inner planet, which also limits by secular interaction the eccentricity growth of the second planet. Notice that this is the case of the Galilean satellites of Jupiter: Io, Europa and Ganymede. In some cases, the eccentricity excitation, although limited, can be sufficient to drive the system unstable.

Particularly interesting in this respect is the work by Bolmont and Mathis (2016) on the coupled evolutions of the stellar spin-rate and planetary orbit. The inner edge of the gas disk, a.k.a. the outer edge of the magnetic cavity, is expected to be at the corotation radius, where the stellar rotation period equals the Keplerian orbital period. Planet migration in the gas-disk should therefore bring the innermost planet at this location. After the removal of the gas, the star is still in the pre-main sequence phase and it undergoes a slow contraction. Consequently, the rotation of the star accelerates, which moves the corotation radius inwards. The tidal interaction with the planet therefore pushes the planet outwards. This situation holds for about $100 \mathrm{My}$. After this time the stellar rotation starts to slow down. The corotation radius moves outwards, typically beyond the planets orbit, so that the planet starts to migrate inwards. If the planet is not alone, this dynamics drives first convergent migration, then divergent migration, with possible interesting consequences for the stability of the system.

\section{Interactions with a stellar companion}

The most well-known consequence of the interaction with a stellar companion is the so-called Lidov-Kozai effect (Lidov, 1962; Kozai, 1962; see Naoz 2016 for a very complete review). A planet initially on a circular orbit, perturbed by an inclined distant star will increase its orbital eccentricity while its orbital plane approaches that of the stellar companion. If the orbit of the stellar companion is circular, this process is reversible, leading to coupled oscillations of the eccentricity and inclination of the planets orbit, driven by the precession of the orbit's argument of perihelion (see the top row of Fig. 4). The reversibility, however, can be broken by the tidal interaction 


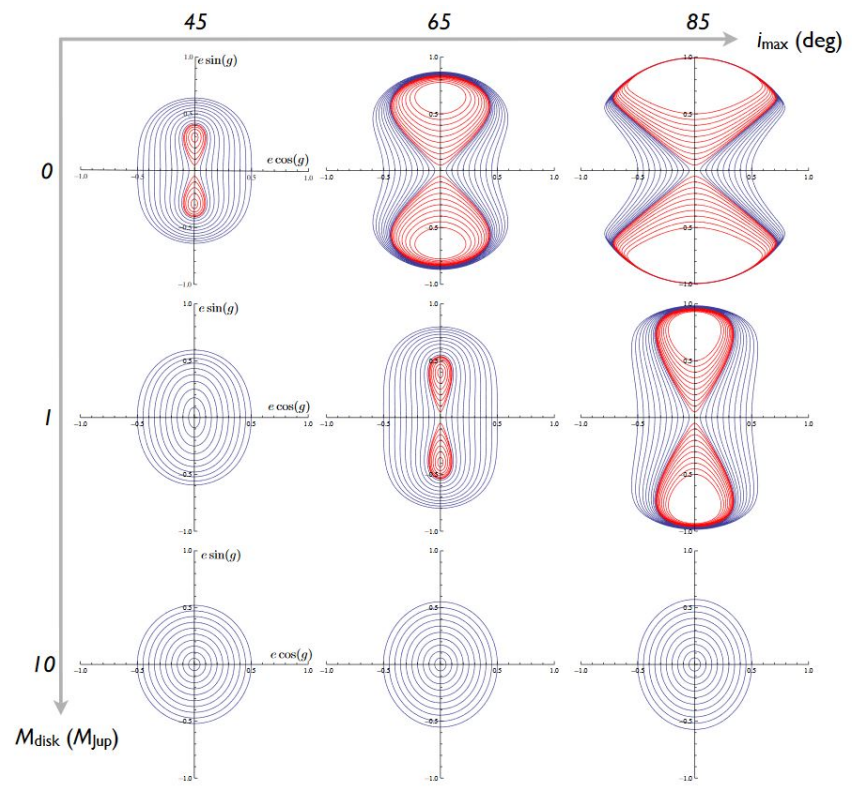

Fig. 4 Dynamical evolution of a planet embedded in a disk under the influence of an inclined stellar companion. The curves in each panel show the cyclic dynamical evolution of the planet in eccentricity $e$ and argument of perihelion $g$. The inclination relative to the stellar orbital plane is anticorrelated with the eccentricity because the quantity $\sqrt{1-e^{2}} \cos (i)$ is conserved. Blue curves have circulating $g$ and red ones librating $g$. The different panels correspond to different inclinations between the orbital plane of the planet at $e=0$ and the stellar companion's orbital plane ( $x$-axis) and different disk masses. Without a disk, an inclined stellar companion (top right panel) induces huge oscillations of the eccentricity of the planet. But with a massive disk (or a planet companion) the stellar perturbation is wiped away and the eccentricity of the planet remains almost constant during a precession cycle of $g$ (bottom right panel). From Batygin et al. (2011).

with the central star; in fact this process has been suggested as responsible for the origin of the so-called hot Jupiters (Fabrycky and Tremaine, 2007). Moreover, if the stellar companion has an eccentric orbit, the evolution of the planet is chaotic and the orbit can flip and become retrograde relative to the central star (e.g. Li et al., 2014).

It should be stressed, however, that only single planets are vulnerable by the Lidov-Kozai effect. The effected is wiped out if the precession rate of the argument of perihelion of a planet induced by a disk or a planet companion is faster than that induced by the stellar companion. Because the stellar companion, although more massive, is typically much farther away, mutual perturbations among planets in a system can easily dominate over the stellar perturbation. A clear example of this is provided by the satellite of Uranus. These satellites orbit on the planet's equatorial plane, and therefore their orbital inclination relative to the orbit of the Sun is 98 degrees. The orbit of a single satellite would therefore be unstable to solar perturbations through the Lidov-Kozai effect, but the satellite system as a whole is stable, 
thanks to the fast precession of the satellites' perihelia induced by mutual perturbations. Similarly, Batygin et al. (2011) showed that a planet embedded in a disk or a system of two giant planets orbiting the central star on coplanar orbits, perturbed by a distant and inclined stellar companion, is likely to be stable against this external perturbation (Fig. 4 , bottom row). However, after the disk is removed, if the planets become unstable for some other reasons and one of the two planets is removed, the remaining planet suddenly starts large eccentricity and inclination oscillations due to the Lidov-Kozai effect. Thus, the Lidov-Kozai effect is the consequence of the instability in a planetary system, rather than the cause.

A different interesting effect produced by a stellar companion has been described by Touma and Sridhar (2015). A strong resonance, called evection, occurs when the precession frequency of the planetary orbit is equal to the orbital frequency of the stellar companion. This resonance excites the orbital eccentricity of the planet. Because in the two-body problem the precession rate of the planets orbit is null, this resonance can exist only in multi-planet systems, where orbital precession are induced by the mutual planetary perturbations. However, the resonance is localized at a specific orbital radius so it is improbable that a given planet is in resonance. Thus, Touma and Sridhar envisioned the intriguing possibility that, in planetary systems undergoing divergent migration due to the planetesimal scattering process, one of the planets may cross the evection resonance, acquire a large eccentricity and destabilize the planetary system as a whole.

\section{Conclusions}

Several observations (reviewed in Sect. 2) suggest that the evolution of a planetary system is not finished when the gas of the protoplanetary disk is removed. The architecture of planetary systems can change profoundly; collisions between planets are possible if the planetary system becomes unstable and the Safronov number is smaller than unity. Both the Solar System and most extrasolar planetary systems have been sculpted by post-gas evolution and planet instabilities.

The cause of these instabilities is still unclear. Planetary systems which are too tightly packed become unstable all by themselves (Chambers et al., 1996; Obertas et al., 2017), without the need of external perturbations (Sect. 3). However, recent numerical simulations show that only $\sim 50 \%$ of the systems of super-Earths generated by gas-driven migration would become unstable this way; instead, fitting the observed distribution requires that the number of systems undergoing post-gas instability exceeds $90 \%$ (Izidoro et al., 2017). Thus, external perturbations should play a role. We discussed several of these perturbations in this chapter. There is probably no universal cause for instability. Several mechanisms can be at play and their relative importance likely depends on a case-to-case basis. The interaction with a remnant planetesimal disk may be the most generic of the processes, and it is the one responsible for the past instability of the giant planets of the Solar System. However, it is not obvious that massive planetesimal populations could have survived at 
the end of the gas-disk phase if the planets had undergone large-range migration in such a disk before.

Future progress might be possible by making statistics of planetary systems architectures for stars in different environments (e.g. binaries, in clusters or isolated, to highlight the importance of external perturbations). But most likely significant progress will occur only with the detection of planets around young stars (say in the first $100 \mathrm{My}$ ) and even still embedded in disks. In fact, so far we only know " mature" planetary systems, for which we need to reconstruct the past evolution with uncertain modeling efforts. The observation of planets around young stars will instead provide direct information on how planetary systems evolve and on which timescale.

\section{Bibliography}

- Adachi I., Hayashi C., Nakazawa K. (1976) The gas drag effect on the elliptical motion of a solid body in the primordial solar nebula. Progress of Theoretical Physics 56:1756-1771

- Adams F.C., Laughlin G., Bloch A.M. (2008) Turbulence Implies that Mean Motion Resonances are Rare. The Astrophysical Journal 683: 1117-1128

- Agnor C.B., Canup R.M., Levison H.F. (1999) On the Character and Consequences of Large Impacts in the Late Stage of Terrestrial Planet Formation. Icarus 142:219-237

- Armitage P.J. (2010) Astrophysics of Planet Formation. Astrophysics of Planet Formation, by Philip J. Armitage, pp. 294. ISBN 978-0-521-88745-8 (hardback). Cambridge, UK: Cambridge University Press, 2010. 294

- Bai X.-N., Stone J. M. (2013) Wind-driven Accretion in Protoplanetary Disks. I. Suppression of the Magnetorotational Instability and Launching of the Magnetocentrifugal Wind. The Astrophysical Journal 769:76

- Batygin K., Morbidelli A., Tsiganis K. (2011) Formation and evolution of lanetary systems in presence of highly inclined stellar perturbers. A\&A 533:A7

- Batygin K., Brown M.E., Betts H. (2012) Instability-driven Dynamical Evolution Model of a Primordially Five-planet Outer Solar System. The Astrophysical Journal 744:L3

- Batygin K., Morbidelli A. (2013) Analytical treatment of planetary resonances. A\&A 556:A28

- Batygin K., Morbidelli A. (2013b) Dissipative Divergence of Resonant Orbits. The Astronomical Journal 145:1

- Batygin K., Adams F.C. (2017) An Analytic Criterion for Turbulent Disruption of Planetary Resonances. The Astronomical Journal 153:120

- Baruteau C., Lin D.N.C. (2010) Protoplanetary Migration in Turbulent Isothermal Disks. The Astrophysical Journal 709:759-773

- Beauge C., Nesvorny D. (2012) Multiple-planet Scattering and the Origin of Hot Jupiters. The Astrophysical Journal 751:119 
- Bitsch B., Johansen A., Lambrechts M., Morbidelli A. (2015) The structure of protoplanetary discs around evolving young stars. A\&A 575:A28

- Bolmont E., Mathis S. (2016) Effect of the rotation and tidal dissipation history of stars on the evolution of close-in planets. Celestial Mechanics and Dynamical Astronomy 126:275-296

- Bonsor A., Raymond S.N., Augereau J.-C., Ormel C.W. (2014) Planetesimaldriven migration as an explanation for observations of high levels of warm, exozodiacal dust. Monthly Notices of the Royal Astronomical Society 441:23802391

- Brasser R., Duncan M.J., Levison H.F. (2007) Embedded star clusters and the formation of the Oort cloud. II. The effect of the primordial solar nebula. Icarus 191:413-433

- Brasser R., Morbidelli A. (2013) Oort cloud and Scattered Disc formation during a late dynamical instability in the Solar System. Icarus 225:40-49

- Capobianco C.C., Duncan M., Levison H.F. (2011) Planetesimal-driven planet migration in the presence of a gas disk. Icarus 211:819-831

- Chambers J.E., Wetherill G.W., Boss A.P. (1996) The Stability of Multi-Planet Systems. Icarus 119:261-268

- Chambers J.E., Wetherill G.W. (1998) Making the Terrestrial Planets: N-Body Integrations of Planetary Embryos in Three Dimensions. Icarus 136:304-327

- Chambers J.E. (2001) Making More Terrestrial Planets. Icarus 152:205-224

- Chatterjee S., Ford E.B., Matsumura S., Rasio F.A. (2008) Dynamical Outcomes of Planet-Planet Scattering. The Astrophysical Journal 686:580-602

- Chatterjee S., Tan J.C. (2014). Inside-out Planet Formation. The Astrophysical Journal 780, 53

- Chatterjee S., Ford E.B. (2015) Planetesimal Interactions Can Explain the Mysterious Period Ratios of Small Near-Resonant Planets. The Astrophysical Journal 803:33

- Chirikov B.V. (1979) A universal instability of many dimensional oscillator systems. Physics Reports, 52: 265

- Cossou C., Raymond S.N., Hersant F., Pierens A. (2014) Hot super-Earths and giant planet cores from different migration histories. A\&A 569:A56

- Cresswell P., Nelson R.P. (2008) Three-dimensional simulations of multiple protoplanets embedded in a protostellar disc. A\&A 482:677-690

- Deck K.M., Payne M., Holman M.J. (2013) First-order Resonance Overlap and the Stability of Close Two-planet Systems. The Astrophysical Journal 774:129

- Deck K.M., Batygin K. (2015) Migration of Two Massive Planets into (and out of) First Order Mean Motion Resonances. The Astrophysical Journal 810:119

- Deienno R., Gomes R.S., Walsh K.J., Morbidelli A., Nesvorny D. (2016) Is the Grand Tack model compatible with the orbital distribution of main belt asteroids?. Icarus 272:114-124

- Deienno R., Morbidelli A., Gomes R.S., Nesvorny D. (2017) Constraining the Giant Planets: Initial Configuration from Their Evolution: Implications for the Timing of the Planetary Instability. The Astronomical Journal 153:153 
- Fabrycky D., Tremaine S. (2007) Shrinking Binary and Planetary Orbits by Kozai Cycles with Tidal Friction. The Astrophysical Journal 669:1298-1315

- Fang J., Margot J.-L. (2012) Architecture of Planetary Systems Based on Kepler Data: Number of Planets and Coplanarity. The Astrophysical Journal 761:92

- Fernandez J.A., Ip W.H. (1984) Some dynamical aspects of the accretion of Uranus and Neptune - The exchange of orbital angular momentum with planetesimals. Icarus 58:109-120

- Ferreira J., Pelletier G. (1995) Magnetized accretion-ejection structures. III. Stellar and extragalactic jets as weakly dissipative disk outflows. A\&A 295:807

- Ford E.B., Rasio F.A. (2008) Origins of Eccentric Extrasolar Planets: Testing the Planet-Planet Scattering Model. The Astrophysical Journal 686:621-636

- Fressin F., Torres G., Charbonneau D., Bryson S.T., Christiansen J., Dressing C.D., Jenkins J. M., Walkowicz L.M., Batalha N.M. (2013) The False Positive Rate of Kepler and the Occurrence of Planets. The Astrophysical Journal 766:81

- Fromang S., Terquem C., Balbus S.A. (2002) The ionization fraction in models of protoplanetary discs. Monthly Notices of the Royal Astronomical Society 329:18-28

- Gammie C.F. (1996) Layered Accretion in T Tauri Disks. The Astrophysical Journal 457:355

- Gladman B. (1993) Dynamics of systems of two close planets. Icarus 106:247

- Goldreich P., Soter S. (1966) Q in the Solar System. Icarus 5:375-389

- Grishin E., Perets H.B. (2015) Application of Gas Dynamical Friction for Planetesimals. I. Evolution of Single Planetesimals. The Astrophysical Journal 811:54

- Hasegawa Y., Pudritz R.E. (2010) Dead Zones as Thermal Barriers to Rapid Planetary Migration in Protoplanetary Disks. The Astrophysical Journal 710:L167L171

- Hasegawa Y., Pudritz R.E. (2011) The origin of planetary system architectures - I. Multiple planet traps in gaseous discs. Monthly Notices of the Royal Astronomical Society 417:1236-1259

- Howard A.W., and 66 colleagues (2012) Planet Occurrence within 0.25 AU of Solar-type Stars from Kepler. The Astrophysical Journal Supplement Series 201:15

- Izidoro A., Ogihara M., Raymond S.N., Morbidelli A., Pierens A., Bitsch B., Cossou C., Hersant F. (2017) Breaking the Chains: Hot Super-Earth systems from migration and disruption of compact resonant chains. Monthly Notices of the Royal Astronomical Society 470:1750-1770

- Johansen A., Davies M.B., Church R.P., Holmelin V. (2012) Can Planetary Instability Explain the Kepler Dichotomy?. The Astrophysical Journal 758:39

- Juric M., Tremaine S. (2008) Dynamical Origin of Extrasolar Planet Eccentricity Distribution. The Astrophysical Journal 686:603-620

- Kirsh D.R., Duncan M., Brasser R., Levison H.F. (2009) Simulations of planet migration driven by planetesimal scattering. Icarus 199:197-209

- Kokubo E., Ida S. (2000) Formation of Protoplanets from Planetesimals in the Solar Nebula. Icarus 143:15-27 
- Kozai Y. (1962) Secular perturbations of asteroids with high inclination and eccentricity. The Astronomical Journal 67:591

- Laughlin G., Steinacker A., Adams F.C. (2004) Type I Planetary Migration with MHD Turbulence. The Astrophysical Journal 608:489-496

- Lega E., Morbidelli A., Nesvorny D. (2013) Early dynamical instabilities in the giant planet systems. Monthly Notices of the Royal Astronomical Society 431:3494-3500

- Levison H.F., Morbidelli A., Gomes R., Backman D. (2007) Planet Migration in Planetesimal Disks. Protostars and Planets V 669-684

- Levison H.F., Morbidelli A., Tsiganis K., Nesvorny D., Gomes R. (2011) Late Orbital Instabilities in the Outer Planets Induced by Interaction with a Selfgravitating Planetesimal Disk. The Astronomical Journal 142:152

- Li G., Naoz S., Holman M., Loeb A. (2014) Chaos in the Test Particle Eccentric Kozai-Lidov Mechanism. The Astrophysical Journal 791:86

- Lidov M.L. (1962) The evolution of orbits of artificial satellites of planets under the action of gravitational perturbations of external bodies. Planetary and Space Science 9:719-759

- Lisse C.M., Wyatt M.C., Chen C.H., Morlok A., Watson D.M., Manoj P., Sheehan P., Currie T.M., Thebault P., Sitko M.L. (2012) Spitzer Evidence for a Lateheavy Bombardment and the Formation of Ureilites in $\eta$ Corvi at $\sim 1$ Gyr. The Astrophysical Journal 747:93

- Lithwick Y., Wu Y. (2012) Resonant Repulsion of Kepler Planet Pairs. The Astrophysical Journal 756:L11

- Liu B., Ormel C.W., Lin D.N.C. (2017) Dynamical rearrangement of superEarths during disk dispersal. I. Outline of the magnetospheric rebound model. Astronomy and Astrophysics 601:A15

- Masset F.S., Morbidelli A., Crida A., Ferreira J. (2006) Disk Surface Density Transitions as Protoplanet Traps. The Astrophysical Journal 642:478-487

- Mayor, M., and 13 colleagues (2011) The HARPS search for southern extra-solar planets XXXIV. Occurrence, mass distribution and orbital properties of superEarths and Neptune-mass planets. ArXiv e-prints arXiv:1109.2497

- Marboeuf U., Bonsor A., Augereau J.-C. (2016) Extrasolar comets: The origin of dust in exozodiacal disks? Planetary and Space Science 133:47-62

- Marino S., and 12 colleagues (2017) ALMA observations of the $\eta$ Corvi debris disc: inward scattering of CO-rich exocomets by a chain of 3-30 Earth mass planets? Monthly Notices of the Royal Astronomical Society 465:2595-2615

- Masset F., Snellgrove M. (2001) Reversing type II migration: resonance trapping of a lighter giant protoplanet. Monthly Notices of the Royal Astronomical Society 320:L55-L59

- Matsumoto Y., Nagasawa M., Ida S. (2012) The orbital stability of planets trapped in the first-order mean-motion resonances. Icarus 221:624-631

- Mills S.M., Fabrycky D.C., Migaszewski C., Ford E.B., Petigura E., Isaacson H. (2016) A resonant chain of four transiting, sub-Neptune planets. Nature 533:509512 
- Morbidelli A., Tsiganis K., Crida A., Levison H.F., Gomes R. (2007) Dynamics of the Giant Planets of the Solar System in the Gaseous Protoplanetary Disk and Their Relationship to the Current Orbital Architecture. The Astronomical Journal 134:1790-1798

- Morbidelli A., Brasser R., Gomes R., Levison H.F., Tsiganis K. (2010) Evidence from the Asteroid Belt for a Violent Past Evolution of Jupiter's Orbit. The Astronomical Journal 140:1391-1401

- Naoz S. (2016) The Eccentric Kozai-Lidov Effect and Its Applications. Annual Review of Astronomy and Astrophysics 54:441-489

- Nesvorny D., Vokrouhlicky D., Morbidelli A. (2007) Capture of Irregular Satellites during Planetary Encounters. The Astronomical Journal 133:1962-1976

- Nesvorny D., Vokrouhlicky D., Morbidelli A. (2013) Capture of Trojans by Jumping Jupiter. The Astrophysical Journal 768:45

- Nesvorny D., Vokrouhlicky D. (2009) Chaotic Capture of Neptune Trojans. The Astronomical Journal 137:5003-5011

- Nesvorny D., Morbidelli A. (2012) Statistical Study of the Early Solar System's Instability with Four, Five, and Six Giant Planets. The Astronomical Journal 144:117

- Nesvorny D. (2015a) Evidence for Slow Migration of Neptune from the Inclination Distribution of Kuiper Belt Objects. The Astronomical Journal 150:73

- Nesvorny D. (2015b) Jumping Neptune Can Explain the Kuiper Belt Kernel. The Astronomical Journal 150:68

- Nesvorny D., Vokrouhlicky D., Dones L., Levison H.F., Kaib N., Morbidelli A. (2017) Origin and Evolution of Short-Period Comets. The Astrophysical Journal $845: 27$

- Obertas A., Van Laerhoven C., Tamayo D. (2017) The stability of tightly-packed, evenly-spaced systems of Earth-mass planets orbiting a Sun-like star. Icarus 293:52-58.

- Ogihara M., Morbidelli A., Guillot T. (2015) A reassessment of the in situ formation of close-in super-Earths. A\&A 578:A36

- Papaloizou J.C.B., Terquem C. (2010) On the dynamics of multiple systems of hot super-Earths and Neptunes: tidal circularization, resonance and the HD 40307 system. Monthly Notices of the Royal Astronomical Society 405:573-592

- Petigura E.A., Howard A.W., Marcy G.W. (2013) Prevalence of Earth-size planets orbiting Sun-like stars. Proceedings of the National Academy of Science 110:19273-19278

- Pichierri G., Morbidelli A., Crida A. (2018) On the Stability of resonant pairs of planets. Celestial Mechanics and Dynamical Astronomy, submitted.

- Pu B., Wu Y. (2015) Spacing of Kepler Planets: Sculpting by Dynamical Instability. The Astrophysical Journal 807:44

- Roig F., Nesvorny D. (2015) The Evolution of Asteroids in the Jumping-Jupiter Migration Model. The Astronomical Journal 150:186

- Safronov V.S. (1972) Evolution of the protoplanetary cloud and formation of the earth and planets. Keter Publishing House, 212 p. 
- Smith A.W., Lissauer J.J. (2009) Orbital stability of systems of closely-spaced planets. Icarus 201:381-394

- Touma J.R., Sridhar S. (2015) The disruption of multiplanet systems through resonance with a binary orbit. Nature 524:439-441

- Tsiganis K., Gomes R., Morbidelli A., Levison H.F. (2005) Origin of the orbital architecture of the giant planets of the Solar System. Nature 435:459-461

- Walsh K.J., Morbidelli A., Raymond S.N., O'Brien D.P., Mandell A.M. (2011) A low mass for Mars from Jupiter's early gas-driven migration. Nature 475:206209

- Wyatt M.C., Smith R., Greaves J.S., Beichman C.A., Bryden G., Lisse C.M. (2007) Transience of Hot Dust around Sun-like Stars. The Astrophysical Journal 658:569-583

- Xu W., Lai D. (2017) Migration of planets into and out of mean motion resonances in protoplanetary discs: analytical theory of second-order resonances. Monthly Notices of the Royal Astronomical Society 468:3223-3238 